\title{
A mobile element-based evolutionary history of guenons (tribe Cercopithecini)
}

\author{
Jinchuan Xing1,4, Hui Wang1, Yuhua Zhang1, David A Ray², Anthony J Tosi3 , \\ Todd R Disotell ${ }^{3}$ and Mark A Batzer*1
}

\begin{abstract}
Address: ${ }^{1}$ Department of Biological Sciences, Biological Computation and Visualization Center, Center for Bio-Modular Multi-scale Systems, Louisiana State University, Baton Rouge, LA 70803, USA, ²Department of Biology, West Virginia University, PO Box 6057, Morgantown, West VA 26506, USA, ${ }^{3}$ Department of Anthropology, New York University, New York, NY 10003, USA and ${ }^{4}$ Department of Human Genetics, University of Utah Health Sciences Center, Salt Lake City, UT 84112, USA

Email: Jinchuan Xing - jxing1@lsu.edu; Hui Wang - hwang7@lsu.edu; Yuhua Zhang - yzhang5@lsu.edu; David A Ray - David.Ray@mail.wvu.edu; Anthony J Tosi - ajt5@nyu.edu; Todd R Disotell - todd.disotell@nyu.edu; Mark A Batzer* - mbatzer@lsu.edu

* Corresponding author
\end{abstract}

Published: 31 January 2007

BMC Biology 2007, 5:5 doi:10.1 186/1741-7007-5-5
Received: 2 October 2006

Accepted: 3I January 2007

This article is available from: http://www.biomedcentral.com/I74I-7007/5/5

(c) 2007 Xing et al; licensee BioMed Central Ltd.

This is an Open Access article distributed under the terms of the Creative Commons Attribution License (http://creativecommons.org/licenses/by/2.0), which permits unrestricted use, distribution, and reproduction in any medium, provided the original work is properly cited.

\begin{abstract}
Background: Guenons (tribe Cercopithecini) are a species-rich group of primates that have attracted considerable attention from both primatologists and evolutionary biologists. The complex speciation pattern has made the elucidation of their relationships a challenging task, and many questions remain unanswered. SINEs are a class of non-autonomous mobile elements and are essentially homoplasy-free characters with known ancestral states, making them useful genetic markers for phylogenetic studies.

Results: We identified I5I novel Alu insertion loci from II species of tribe Cercopithecini, and used these insertions and 17 previously reported loci to infer a phylogenetic tree of the tribe Cercopithecini. Our results robustly supported the following relationships: (i) Allenopithecus is the basal lineage within the tribe; (ii) Cercopithecus lhoesti (L'Hoest's monkey) forms a clade with Chlorocebus aethiops (African green monkey) and Erythrocebus patas (patas monkey), supporting a single arboreal to terrestrial transition within the tribe; (iii) all of the Cercopithecus except $C$. Ihoesti form a monophyletic group; and (iv) contrary to the common belief that Miopithecus is one of the most basal lineages in the tribe, $M$. talapoin (talapoin) forms a clade with arboreal members of Cercopithecus, and the terrestrial group ( $C$. Ihoesti, Chlorocebus aethiops and $E$. patas) diverged from this clade after the divergence of Allenopithecus. Some incongruent loci were found among the relationships within the arboreal Cercopithecus group. Several factors, including incomplete lineage sorting, concurrent polymorphism and hybridization between species may have contributed to the incongruence.
\end{abstract}

Conclusion: This study presents one of the most robust phylogenetic hypotheses for the tribe Cercopithecini and demonstrates the advantages of SINE insertions for phylogenetic studies. 


\section{Background}

Guenons (tribe Cercopithecini) are a species-rich group of primates with a distribution throughout sub Saharan Africa. With their diverse morphology, ecology, behavior and social organizations, guenons have attracted considerable attention from both primatologists and evolutionary biologists $[1,2]$. In addition, some species in the tribe (e.g. Chlorocebus aethiops) have been widely used in biomedical studies [3-5]. Based on Groves' classification [6], the tribe Cercopithecini consists of five genera (Erythrocebus, Chlorocebus, Cercopithecus, Miopithecus and Allenopithecus) comprising 36 species. The evolutionary history of guenons may include a rapid basal radiation, and it is very likely that speciation is an ongoing process within the tribe $[1,7]$. This complex speciation pattern has made the elucidation of the species relationships a challenging task, and many questions remain unanswered about the tribe composition (e.g. inclusion of Allenopithecus in the tribe), genera definitions (e.g. whether to consider Chlorocebus as a separate genus) and in particular, the phylogenetic relationships among genera and species $[6,8,9]$.

To elucidate the phylogeny of tribe Cercopithecini, several molecular studies have been conducted, and some aspects of their phylogeny have been inferred with a degree of confidence [7,10-14]. Nevertheless, two major questions remain unanswered:

- (i) is genus Cercopithecus paraphyletic and what are the species relationships within the genus?

- (ii) what is the branching order of the major groups?

Regarding the first question, Cercopithecus was previously considered to be a monophyletic group [10], but recent molecular [7,13,14] and karyotype studies [11] support grouping of the terrestrial taxa, Cercopithecus lhoesti species group, Chlorocebus and Erythrocebus, into a single lineage. This topology supports a single arboreal to terrestrial transition within the tribe, and divides the current genus Cercopithecus into a paraphyletic group. For the phylogenetic relationships among the arboreal members of Cercopithecus, several types of molecular data, including $\mathrm{X}$ and $\mathrm{Y}$ chromosome DNA sequences, protein sequences and karyotypes, recognize a clade containing a cephus species group and a mitis group. X chromosome DNA and protein studies also support a neglectus + diana + mona group clade (see summary in Tosi et al [7]). Nevertheless, a robust phylogenetic hypothesis of lower-level relationships among arboreal Cercopithecus species is not yet confirmed, as there is discordant evidence $[7,9]$.

The other major remaining question is the relationships between the major groups within Cercopithecini. It is thought that the tribe can be divided into four major clades: an arboreal Cercopithecus clade, a terrestrial clade (C. Ihoesti species group, Chlorocebus and Erythrocebus), Miopithecus and Allenopithecus. Miopithecus and Allenopithecus are usually considered to be the most basal lineages of the tribe $[11,15,16]$. Although recent studies $[7,14]$ have provided robust support for the most basal lineage position of Allenopithecus, the relationships of the other three major clades (i.e. the arboreal Cercopithecus clade, the terrestrial clade, and Miopithecus) remain unclear. A set of molecular markers that are independent of DNA sequence data may help to resolve these phylogenetic questions in guenons.

Short interspersed element (SINE) insertions are a class of retrotransposons that integrate into a genome via an RNA intermediate [17]. SINE-based phylogenetic systems were put into practice in the early 1990 s $[18,19]$, and certain unique characteristics make them particularly promising for evolutionary analyses [20-23]. Briefly, SINEs are usually unidirectional characters, with the absence of the insertion being the ancestral state. Precise removal of SINEs is extremely rare after their fixation in the genome, and is very unlikely to happen in multiple genomes [24]. In addition, SINE-based analysis does not rely directly on analyzing DNA sequence data, which makes SINEs an independent complement to traditional DNA-based molecular studies that focus on sequence substitutions. In recent years, SINE-based phylogenetic analysis has proven to be a powerful tool for various levels of phylogenetic studies, and many controversial phylogenetic relationships that could not be solved using traditional molecular data have been successfully elucidated [21-23].

Alu elements are the most successful SINEs in primate genomes in terms of copy number. During primate evolution, Alu elements have proliferated in all primates and expanded to more than one million copies in the human genome $[25,26]$. Several studies have been conducted to infer primate phylogenetic relationships using Alu elements, including the human, chimpanzee and gorilla trichotomy [27], Old World [28] and New World monkey phylogeny [29], tarsier affiliation [30], and strepsirrhine phylogeny [31].

Using a combination of computational data-mining and PCR-based display methods, we identified $168 \mathrm{Alu}$ insertion loci, which contained a total of 179 Alu insertions that had integrated into 11 cercopithecine genomes at various times. These insertions were used to construct a robustly supported phylogenetic hypothesis for the tribe Cercopithecini. The major relationships are: (i) Allenopithecus is the basal lineage of the tribe; (ii) C. Ihoest $i$ (L'Hoest's monkey) forms a clade with Chlorocebus aethiops (African green monkey) and E. patas (patas monkey), supporting a single transition to a terrestrial lifestyle; (iii) 
all Cercopithecus species except C. lhoesti form a monophyletic group; and (iv) contrary to the usual hypothesis that Miopithecus is one of the most primitive lineages in the tribe, our results suggest that the divergence between the arboreal Cercopithecus group and M. talapoin happened after the divergence of the terrestrial group.

\section{Results}

In total, 168 loci were selected for the phylogenetic analysis. Of these, 144 were identified using PCR display methodology, 7 were collected from available African green monkey (Chlorocebus aethiops) genomic sequences using a computational data mining approach (see Methods for details) and 17 loci were selected from a previous study [28]. All loci were genotyped on a primate panel composed of 12 Old World primate species (Table 1), including 11 species within tribe Cercopithecini, with Pygathrix nemaeus (red-shanked Douc langur) as the outgroup. Gel electrophoresis results of five amplifications are shown in Figure 1. For the potential informative loci (i.e. loci in which more than one but not all species showed Alu insertion-sized amplicons), all of the PCR amplicons that appeared to have Alu insertions were sequenced to verify the presence of the Alu elements.

The sequencing verification recovered 11 loci that contained adjacent independent insertion events (i.e. independent insertions in different species that are within sufficiently close genomic proximity to be amplified by a single set of PCR primers; see Discussion for details). These adjacent independent insertion events were treated as independent markers in the subsequent analysis. Overall, 179 markers were used for the phylogenetic analysis. For presence/absence characters in which the absence of the marker is assumed to be the ancestral state, Dollo parsimony is the most appropriate analysis. Therefore, we implemented an exhaustive search using Dollo parsimony, designating $P$. nemaeus as an outgroup taxon. The analysis indicated that 78 loci were parsimony informative and resulted in two most parsimonious trees; the strict consensus for these two trees is shown in Figure 2A (219 steps, consistency index $=0.817$; homoplasy index $=$ 0.183 ; retention index $=0.802$ ). A likelihood test for every branch [32] and 10000 bootstrap replicates were performed. The significance level and the percentage of bootstrap replicates supporting each branch are indicated. A collapsed version of this tree is shown (Figure 2B), in which branches that did not garner statistically significant support based on the likelihood test were collapsed into polytomies.

\section{The monophyly of the terrestrial group}

We identified 10 Alu insertions shared by C. lhoesti, Chlorocebus aethiops and $E$. patas. This result strongly supported $(p<0.001)[32]$ the monophyly of the terrestrial group and the paraphyly of the previously defined genus Cercopithecus. This result is congruent with the single transition hypothesis, which states that there was only one transition from an arboreal to terrestrial lifestyle within the tribe $[7,14,33]$. Unfortunately, we did not identify enough informative markers to gain significant support for the relationships between taxa within the terrestrial group.

\section{The branching order of the major clades}

Given the unambiguous clustering of a single terrestrial clade, our results support the division of the tribe Cercopithecini into four major clades: the arboreal Cercopithecus species, a terrestrial clade, Miopithecus, and Allenopithecus. Six Alu insertions were shared by all taxa except A. nigroviridis. This unambiguously placed Allenopithecus as the basal lineage within the tribe $(p<0.01)$. Another seven insertions joined M. talapoin to all arboreal Cercopithecus

Table I: DNA samples of all species examined in this study.

\begin{tabular}{llll}
\hline Species Names & Common Names & Origin & ID number \\
\hline Allenopithecus nigroviridis & Allen's swamp monkey & IPBIR* & PR00I98 \\
Cercopithecus ascanius & Red-tailed monkey & IPBIR* & PR00566 \\
Cercopithecus cephus & Mustached guenon & IPBIR* & PR0053I \\
Cercopithecus diana & Diana monkey & Tosi & N/A \\
Cercopithecus lhoesti & L'Hoest's monkey & IPBIR* & PR00283 \\
Cercopithecus nictitans & White-nosed guenon & Tosi & N/A \\
Cercopithecus petaurista & Lesser white-nosed monkey & IPBIR* & PR00762 \\
Cercopithecus wolfi wolfi & Wolf's guenon & IPBIR* & PR00486 \\
Chlorocebus aethiops & African green monkey & ATCC $\ddagger$ & CCL70 \\
Erythrocebus patas & Patas monkey & SDFZ & KB5435 \\
Miopithecus talapoin & Talapoin & SDFZ & OR 755 \\
Pygathrix nemaeus & Red-shanked Douc langur & SDFZ & OR 259
\end{tabular}

* Integrated Primate Biomaterials and Information Resource, http://www.ipbir.org.

† Blood sample from Dr. Anthony Tosi.

$\ddagger$ Cell line provided by the American Type Culture Collection.

$\S$ Frozen Zoo ${ }^{\circledR}$, San Diego Zoo, http://conservationandscience.org. 
A)

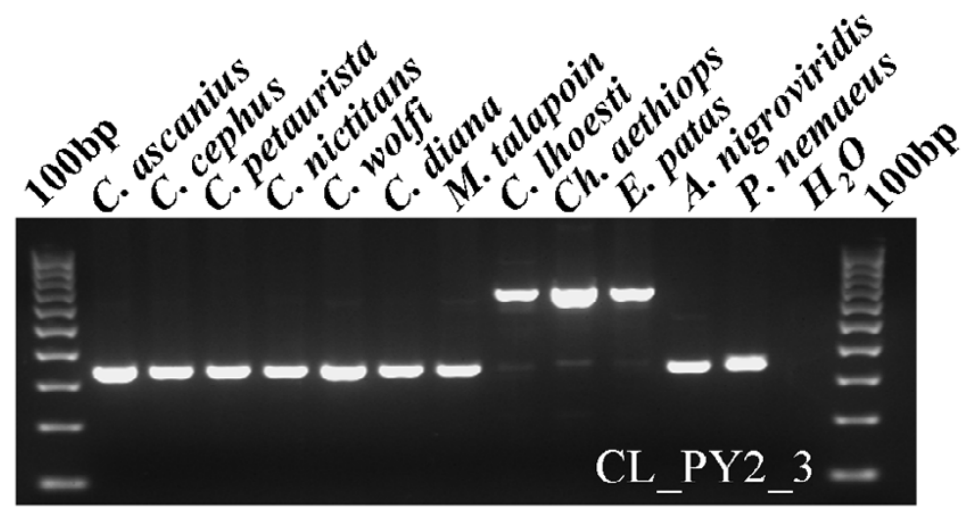

B)

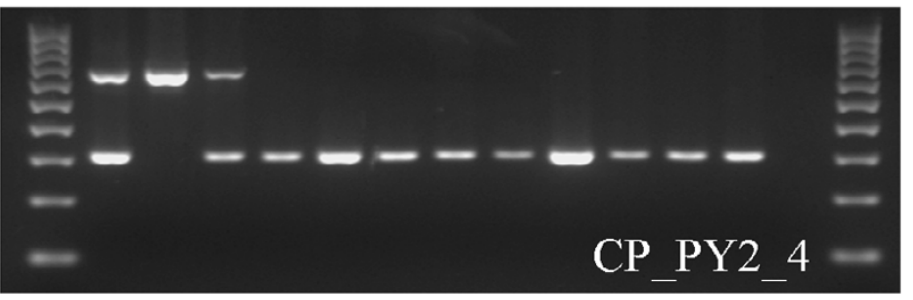

C)

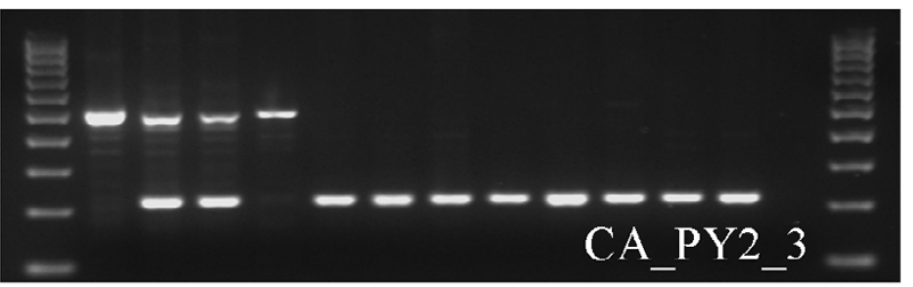

D)

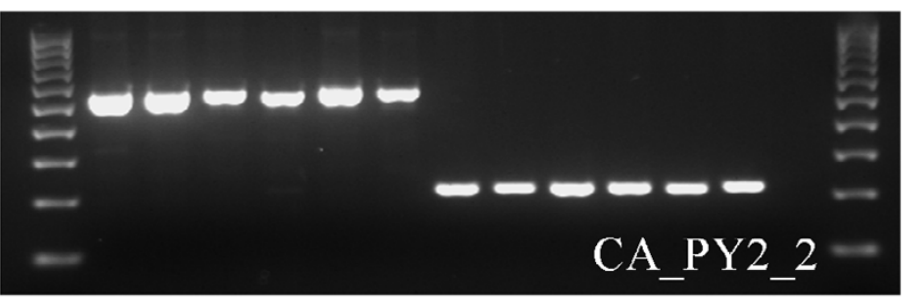

E)

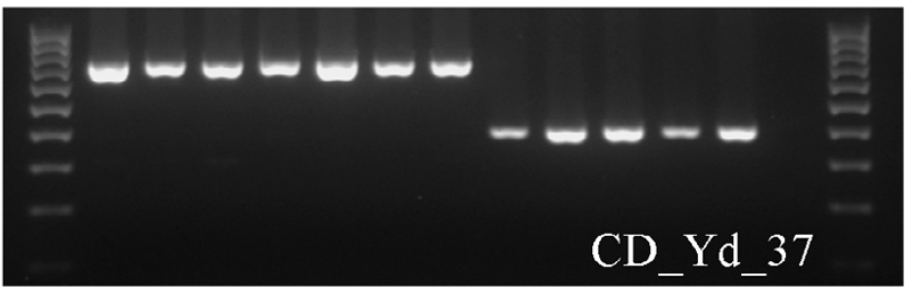

\section{Figure I}

Phylogenetic analysis of Alu insertion polymorphisms in guenon genomes. Gel electrophoresisresults of five amplifications used to determine the phylogenetic origin of individual Alu insertions in Cercopithecini species are shown. Upper DNA fragments indicate "filled" sites where Alu elements have inserted, and lower fragments indicate "empty" sites containing no Alu insertion. The DNA template used in each reaction and the locus designation are shown. (A) An Alu insertion specific for terrestrial guenons (C. Ihoesti, Chlorocebus aethiops and E. patas); (B) An Alu insertion restricted to the cephus species group (C. ascanius, $C$. cephus and C. petaurista); (C) An Alu insertion clustering the cephus species group and C. nictitans; (D) An Alu insertion restricted the arboreal Cercopithecus group; (E) An Alu element present in the arboreal Cercopithecus group and M. talapoin. 

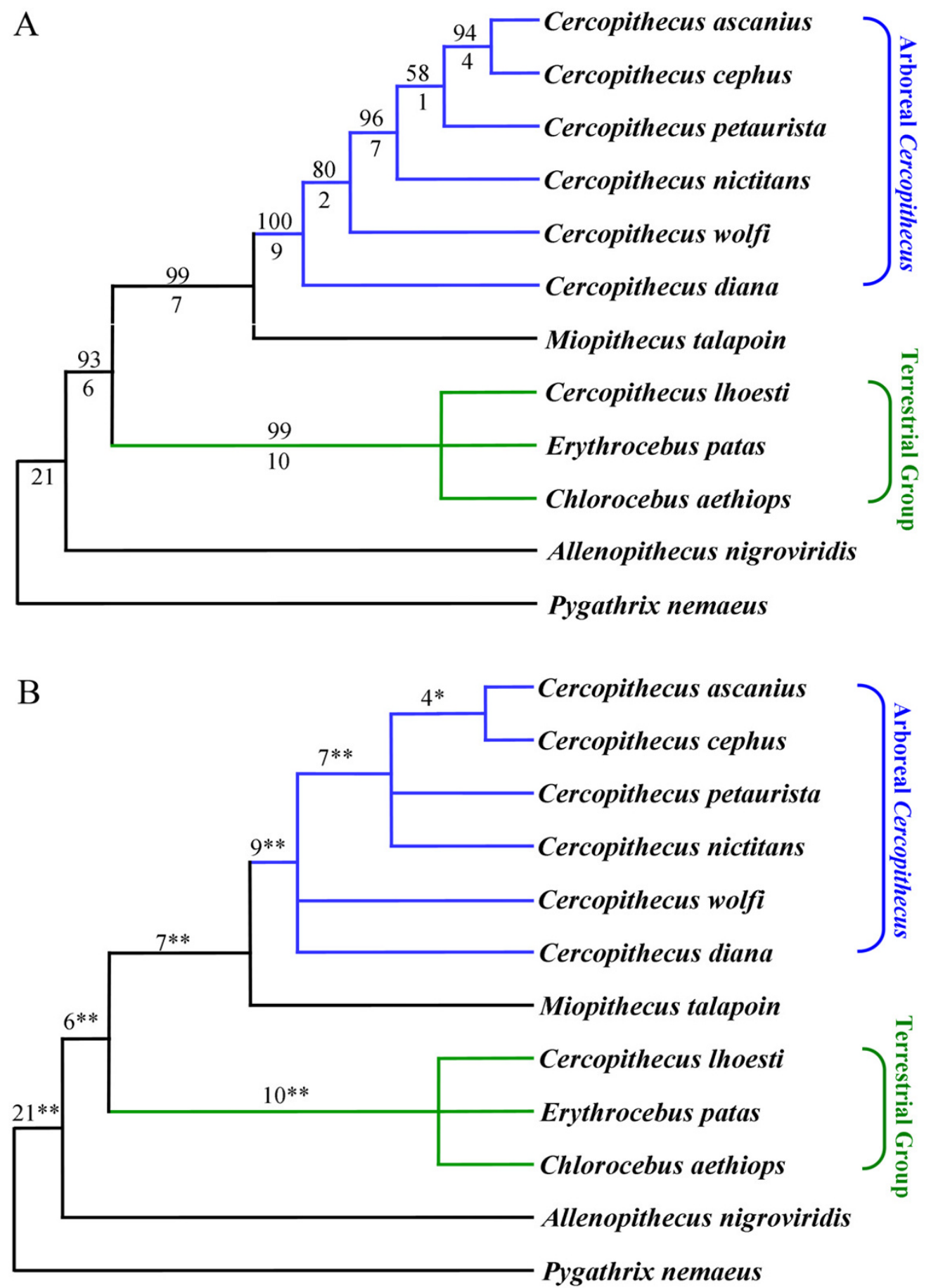

Figure 2

Cladograms of tribe Cercopithecini phylogenetic relationships derived from Alu insertion polymorphisms. The blue section of each phylogeny indicates species of Cercopithecus with an arboreal lifestyle and the green section indicates species with a terrestrial lifestyle. (A) The strict consensus of the two most parsimonious trees from analysis of I79 Alu insertion polymorphisms. The numbers above the branches indicate the percentage of bootstrap replicates (I0000 iterations) producing trees that include that node. Numbers below the branches indicate the numbers of unambiguous insertions supporting each node. (B) The collapsed version of tree A. Branches that did not gain statistically significant support in the likelihood test [32] were collapsed into polytomies. The significance level of each node is indicated by either $*(p<0.05)$ or $* *(p<0.0 \mathrm{I})$. 
species (i.e. C. ascanius, C. cephus, C. petaurista, C. wolfi, C. diana and $C$. nictitans). These results suggest that the terrestrial group diverged after Allenopithecus but before the separation of Miopithecus from the arboreal Cercopithecus species $(p<0.01)$. The divergence of Miopithecus and the monophyly of the six arboreal Cercopithecus species were supported by nine Alu insertion loci present in all arboreal taxa but not in Miopithecus $(p<0.01)$.

\section{The relationships among the arboreal Cercopithecus species}

Of the six arboreal Cercopithecus species we tested, two clades were well supported. C. ascanius and C. cephus were clustered together to the exclusion of $C$. petaurista, based on four $A l u$ insertions $(p<0.05)$. The $C$. ascanius and $C$. cephus clade was linked to C. petaurista and C. nictitans by seven insertions to form a polytomy $(p<0.01)$. Despite the relatively strong support for these two nodes, we should note that there were loci that supported alternative topologies among the arboreal Cercopithecus species. For example, when the affiliation of $C$. diana is considered, $A l u$ insertions present in $C$. diana suggested at least four alternative phylogenetic hypothesis (Figure 3). Two Alu insertions (TA26 and CD_Yd_40) grouped C. diana with C. ascanius, C. cephus and C. petaurista, but not C. nictitans. In contrast, two other Alu elements (CD_Yd_27 and CN_PY2_53A) were found only in C. diana and C. nictitans. In addition, one marker (CD_PY2_36) supported the grouping of $C$. diana, $C$. nictitans and C. cephus, and one marker (CD_PY2_41) grouped C. diana, C. wolfi and C. petaurista together. Owing to this incongruence and the small number of makers supporting each hypothesis, none of these hypotheses gained statistically significant support using the likelihood test.

Another noteworthy result is that a number of $A l u$ insertion loci appeared to be heterozygous in certain species (e.g. both a filled band and an empty band are present) (Figures $1 \mathrm{~B}$ and 1C). Several mechanisms, including incomplete lineage sorting, concurrent polymorphism and introgression may have contributed to this finding.

\section{Discussion \\ The placement of Allenopithecus and Miopithecus}

Our results indicate that Allenopithecus is the most basal lineage of the tribe. A monophyletic terrestrial clade comprising C. lhoesti, E. patas, and Chlorocebus aethiops was the next to diverge from other guenons after the split of Allenopithecus, and Miopithecus subsequently diverged from the rest of arboreal Cercopithecus. This placement of Miopithecus represents a major difference compared with other phylogenetic analyses of the Cercopithecini. Despite the general view that Miopithecus may share some primitive features of the group (e.g. sexual swelling), no studies to date have provided robust support for a definitive placement for Miopithecus. For example, in a study using Y-chromosome DNA data [14], a four-way polytomy for Allenopithecus; Miopithecus, a terrestrial clade and an arboreal Cercopithecus clade was obtained. Studies using Xchromosome sequences identified Allenopithecus as the most basal lineage of the tribe, although a three-way polytomy consisting of Miopithecus, the terrestrial clade and the arboreal Cercopithecus clade persisted [7,14]. Our results represent the first statistically significant support for the clustering of Miopithecus with the arboreal Cercopithecus clade.

\section{Phylogenetic relationship of the arboreal Cercopithecus species}

Owing to rapid speciation and the resulting close relationships within the genus Cercopithecus, a detailed lower-level hypothesis of relationships has been lacking. To date, the only robustly supported relationships are the separation of a cephus group + mitis group clade, and a neglectus + diana + mona group clade $[7,14]$. In the current study, several relationships within the genus are supported by Alu insertions that have statistical support. For example, the clustering of C. ascanius and C. cephus within the cephus group (represented in this study by C. ascanius; C. cephus and $C$. petaurista) is well supported. The close relationship between the mitis group (represented here by C. nictitans) and the cephus group is also strongly supported. The diana group (represented here by $C$. diana) appears to be basal within the arboreal Cercopithecus clade. However, this hypothesis deserves further investigation, owing to the relatively low level of support for the placement of $C$. wolfi. Overall, these results are in good agreement with previous studies using $\mathrm{X}$ and $\mathrm{Y}$ chromosome DNA data, and provide robust independent evidence for the suggested relationships $[7,14]$.

\section{Arboreal to terrestrial lifestyle transition}

Our results provide strong support for a single evolutionary transition from arboreality to terrestriality within the tribe Cercopithecini. The inference of a single transition to a ground-dwelling lifestyle suggests that such changes in substrate preference are probably rare in the history of the primates and therefore may lend greater significance to a similar change at the beginning of the hominin radiation. In addition, the guenon transition to a terrestrial lifestyle is estimated to have occurred at the Miocene/Pliocene boundary [7]. This is significant because the same time window brackets the origins of hominin terrestrial bipedalism. Thus, similar factors may have driven early human ancestors and the terrestrial guenon progenitor to a largely ground-dwelling way of life.

\section{Incongruent patterns of Alu insertions among lineages}

There are three major scenarios in SINE-based phylogenetic analyses that can lead to confounding results: adja- 


\section{Alternative \#1}

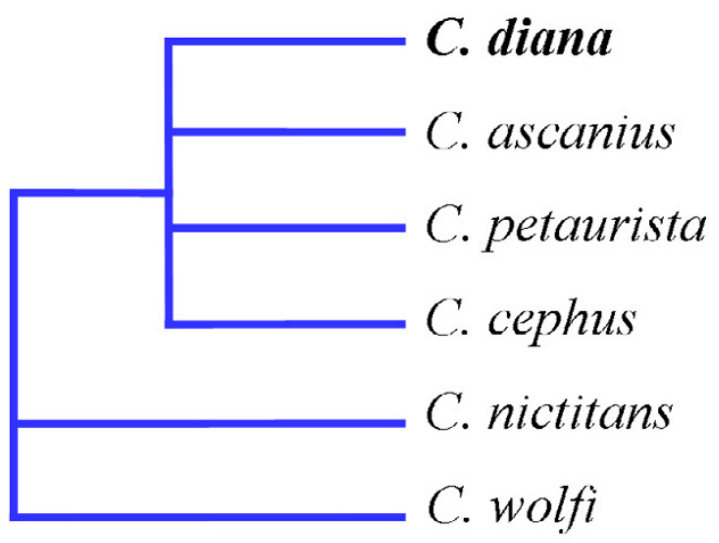

Alternative \#3

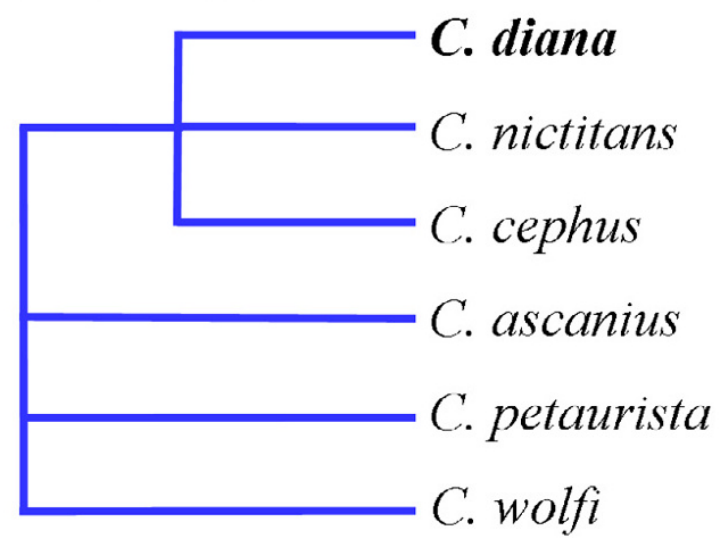

Alternative \#2

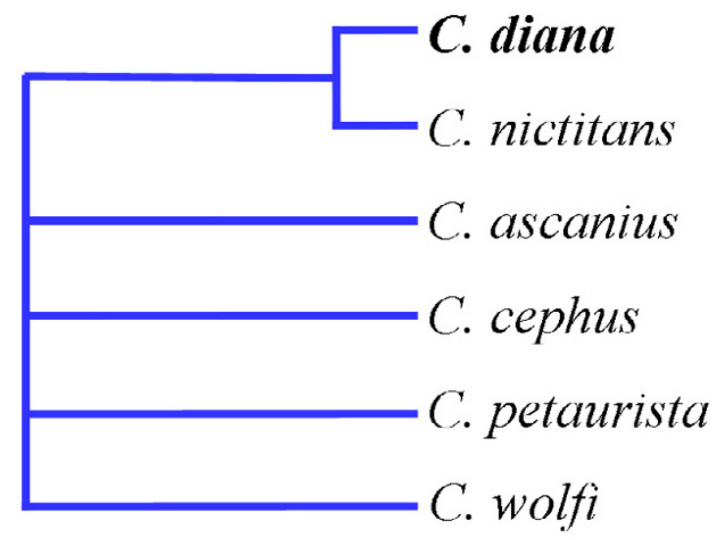

Alternative \#4

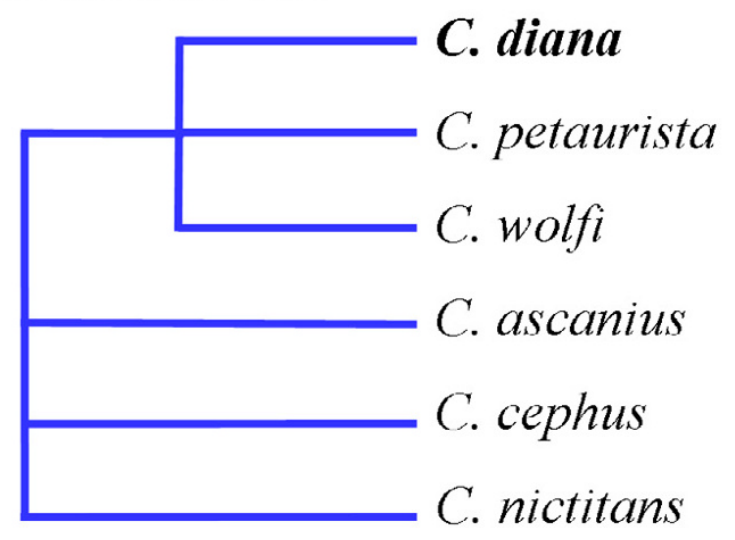

\section{Species}

CD

CN

CA CC CP CW

\section{Alternative \#1}

TA26

CD_Yd 40

\section{Alternative \#2}

CD_Yd 27

CN_PY2 53A

\section{Alternative \#3}

CD_PY2_36

\section{Alternative \#4}

CD PY2 41

$\begin{array}{llllll}1 & 0 & 1 & 1 & 1 & 0 \\ 1 & 0 & 1 & 1 & 1 & 0\end{array}$

$\begin{array}{llllll}1 & 1 & 0 & 0 & 0 & 0 \\ 1 & 1 & 0 & 0 & 0 & 0\end{array}$

$\begin{array}{llllll}1 & 1 & 0 & 1 & 0 & 0\end{array}$

$\begin{array}{llllll}1 & 0 & 0 & 0 & 1 & 1\end{array}$

Figure 3

Alu insertions support different phylogenetic hypotheses for the affiliation of $C$. diana. Four alternative hypotheses and the markers supporting each hypothesis are shown. Presence of the insert was coded as "I" and absence of the insert as "0". CD, C. diana; CN, C. nictitans; CP, C. petaurista; CA, C. ascanius; CC, C. cephus; CW, C. wolfi. 
cent independent insertions, incomplete lineage sorting, and hybridization between species. Adjacent independent insertions refer to the scenario that in different species, different Alu elements are inserted in close genomic proximity and are therefore amplified within the same PCR amplicon during genotyping. In this study, we sequenced all informative loci to confirm that the appropriately size amplicon contained the same Alu insertions, and all loci containing parallel independent insertions have been excluded or treated as independent markers (see Results section). Therefore, the incongruence observed in our current dataset cannot be explained by invoking this explanation.

Incomplete lineage sorting is caused by alternative fixation/elimination of ancestral polymorphisms in populations of daughter lineages. Observations of incomplete lineage sorting can be particularly problematic when examining taxa that have undergone rapid bursts of speciation [22,34]. It is believed that the tribe Cercopithecini diverged from tribe Papionini about 11 million years ago $[7,35]$. However, owing to the lack of fossil evidence, the divergence times of subsequent groups are less certain. For the arboreal Cercopithecus clade, multiple speciation events may have occurred during a very short period to create the six species groups, containing the $>20$ species we see today [1]. If that is the case, many polymorphic Alu elements present in the guenon ancestral population have the potential to remain polymorphic before, during, and after these speciation events. These insertions were eventually fixed in or lost from the genomes of daughter species, and may have resulted in the incongruent insertion presence/absence patterns observed.

Finally, occasional introgressions via hybridization present yet another hypothesis to explain the incongruent Alu patterns. The ranges of closely related species within many guenon species groups often overlap geographically, and many of these species are known to hybridize with each other. For example, it is known that hybridizations occur between the red-tailed monkey group (cephus species group) and the blue monkey group (mitis species group, represented here by $C$. nictitans) in their contact zones [36]. These hybridizations may have contributed to the polytomy of C. ascanius, C. cephus, C. petaurista (the cephus species group) and $C$. nictitans (the mitis species group) in our study. In fact, introgression may even have introduced certain $\mathrm{Alu}$ insertion loci into species other than the arboreal Cercopithecus clade and provide a plausible explanation for some of the incongruent results in our study.

\section{Conclusion}

Based on 179 Alu insertions, a robust phylogenetic hypothesis has been constructed and the placement for
Miopithecus is inferred with statistically significant support for the first time. In addition, our observations indicate that the group of arboreal Cercopithecus species may have experienced a series of rapid, even simultaneous, speciation events, and thus a number of Alu insertions have been randomly fixed in different lineages. Hybridization between different species in the tribe may have also contributed to the complex patterns in our dataset. Owing to the unidirectional insertion property and extremely low removal rate of fixed SINE insertions, these markers can be used to construct phylogenetic hypothesis with high statistical power and provide evidence independent to sequence-based molecular phylogenetic studies.

\section{Methods}

\section{Computational data mining}

Genomic sequences from Chlorocebus aethiops(African green monkey) were obtained from the NIH Intramural Sequencing Center [37], as part of the Comparative Vertebrate Sequencing Initiative. The sequences were broken into 10000-bp fragments and compared with the human genome (hg18) and the rhesus monkey genome (rheMac2) using the BLAST-Like Alignment Tool (BLAT) [38]. Fragments containing insertion/deletions were extracted and annotated to identify putative lineage-specific Alu insertions using previously reported methods [39]. In order to allow a focus on the cercopithecine lineage, only Alu elements present in the Chlorocebus aethiops sequence but absent in the orthologous regions of the rhesus monkey genome were excised along with $1000 \mathrm{bp}$ of flanking sequence in both directions. Flanking oligonucleotide primers for PCR amplification of each Alu element were then designed using Primer3 software [40]. The primers were subsequently screened against the GenBank NR database using the Basic Local Alignment Search Tool (BLAST) program [41] to determine if they resided in unique DNA sequences.

\section{PCR display methodology and PCR genotyping}

The Alu element PCR-display methodology and Alu-specific primers described by Xing et al [28] were used for identifying species-specific Alu insertions. To prevent ascertainment bias, every cercopithecine species in this study was subject to this methodology and Alu insertion loci were identified from all species. Oligonucleotide primer pairs were initially tested using Chlorocebus aethiops DNA templates with a temperature gradient PCR (48$60^{\circ} \mathrm{C}$ ) to determine the most appropriate annealing temperature for further analysis. All loci were screened against a primate panel that was composed of DNA samples from 12 Old World primate species (Table 1). Because the quantity of genomic DNA samples for most species is limited, all samples except Chlorocebus aethiops were subjected to whole genome amplification using the GenomiPhi genome amplification kit (Amersham, Sunnyvale, CA, 
USA) following the manufacturer's instructions. The amplified samples were then purified and divided into aliquots for locus-specific PCR analysis.

PCR amplification of each locus was performed as described by Xing et al [28]. The resulting PCR products were run on $2 \%$ agarose gels with $0.25 \mu \mathrm{g}$ of ethidium bromide and visualized using ultraviolet fluorescence. Detailed information for each locus including primer sequences, annealing temperature, PCR product sizes, chromosomal locations and amplification results are available on our website (supplemental table. located under "Publications") [42].

\section{DNA sequence analysis}

For the potentially informative loci, all PCR amplicons that appeared to have Alu insertions were subjected to sequence analysis to verify the presence of the Alu elements. Selected taxa from several uninformative loci were also sequenced to confirm the presence of the Alu elements. Individual PCR products were either directly sequenced or were cloned and sequenced as described previously [29]. Sequences for each locus were aligned against rhesus monkey (when available) or human orthologous sequence obtained via the BLAT search. Sequence alignments of these loci are available from our website (supplemental table. located under "Publications") [42]. The DNA sequences generated for this project have been deposited in GenBank (accession numbers DQ977747-DQ978210).

\section{Phylogenetic analysis}

Alu insertion loci were included in phylogenetic analysis if amplicons were generated in at least seven out of the 11 cercopithecine taxa in our panel, and only 2 distinct classes of amplicons were obtained (i.e. Alu filled size and pre-integration or Alu empty size). Any primer pair that generated multiple paralogous fragments across the panel was excluded from the analysis.

We implemented an exhaustive search in PAUP* software (version 4.0b10) [43] using Dollo parsimony and designating the Pygathrix nemaeus as an outgroup taxon. Presence of the insert was coded as "1" and absence of the insert as " 0 ". If no amplification was observed for a given locus in any taxon, the character state was coded as unknown ("?"). For loci containing adjacent independent insertion events, the independent insertions were treated as independent markers. In total, 10000 bootstrap replicates were performed on the data. A statistical test for evaluating SINE insertions based on a likelihood model [32] was also performed to assess the statistical significance of each branch of the resulting tree.

\section{Authors' contributions}

JX, AJT, TRD and MAB designed the study. JX, HW, YZ and DAR performed the experiments. JX and HW analyzed the dataset. MAB directed the project. All authors were involved in preparing the manuscript.

\section{Acknowledgements}

We thank Dr Robb T. Brumfield, Dr Miriam Konkel and Thomas J. Meyer for their useful comments and critical readings during the preparation of the manuscript. This research was supported by the National Science Foundation grants BCS-02 I8338 (MAB) and EPS-03464I I (MAB), National Institutes of Health GM59290 (MAB), and the State of Louisiana Board of Regents Support Fund (MAB). DAR was supported by the Eberly College of Arts and Sciences at WVU.

\section{References}

I. Butynski TM: The guenons: An overview of diversity and taxonomy. In The Guenons: Diversity and Adaptation in African Monkeys Edited by: Glenn ME, Cords M. New York: Kluwer Academic Publishers; 2002:3-13.

2. Butynski TM: Conservation of the guenons: an overview of status, threats, and recommendations. In The Guenons: Diversity and Adaptation in African Monkeys Edited by: Glenn ME, Cords M. New York: Kluwer Academic Publishers; 2002:79-97.

3. Broussard SR, Staprans SI, White R, Whitehead EM, Feinberg MB, Allan JS: Simian immunodeficiency virus replicates to high levels in naturally infected African green monkeys without inducing immunologic or neurologic disease. Journal of virology 200I, 75(5):2262-2275.

4. Lee AM, Miksys S, Palmour R, Tyndale RF: CYP2B6 is expressed in African Green monkey brain and is induced by chronic nicotine treatment. Neuropharmacology 2006, 50(4):44I-450.

5. Carlsson HE, Schapiro SJ, Farah I, Hau J: Use of primates in research: a global overview. American journal of primatology 2004, 63(4):225-237.

6. Groves C: Primate Taxonomy Washington, D.C: Smithsonian Press; 2001.

7. Tosi AJ, Detwiler KM, Disotell TR: X-chromosomal window into the evolutionary history of the guenons (Primates: Cercopithecini). Mol Phylogenet Evol 2005, 36(I):58-66.

8. Groves CP: The phylogeny of the Cercopithecoidea. In Old World Monkeys Edited by: Whitehead PF, Jolly CJ. Cambridge: Cambridge University Press; 2000:77-98.

9. Disotell TR: Molecular systematics of the Cercopithecidae. In Old World Monkeys Edited by: Whitehead PF, Jolly CJ. Cambridge: Cambridge University Press; 2000:29-56.

10. Ruvolo M: Genetic evolution in the African guenons. In A Primate Radiation: Evolutionary Biology of the African Guenons Edited by: Gautier-Hion A, Bourliere F, Gautier JP, Kingdon J. New York: Cambridge University Press; 1988:439-45I.

II. Dutrillaux B, Muleris M, Gouturier J: Chromosomal evolution of Cercopithecinae. In A Primate Radiation: Evolutionary Biology of the African Guenons Edited by: Gautier-Hion A, Bourliere F, Gautier JP, Kingdon J. New York: Cambridge University Press; I 988: I 50-I59.

12. Disotell TR, Raaum RL: Molecular timescale and gene tree incongruence in the guenons. In The Guenons: Diversity and Adaptation in African Monkeys Edited by: Glenn ME, Cords M. New York: Kluwer Academic Publishers; 2002:25-34.

13. Tosi AJ, Disotell TR, Morales JC, Melnick DJ: Cercopithecine Ychromosome data provide a test of competing morphological evolutionary hypotheses. Mol Phylogenet Evol 2003, 27(3):510-52I.

14. Tosi AJ, Melnick DJ, Disotell TR: Sex chromosome phylogenetics indicate a single transition to terrestriality in the guenons (tribe Cercopithecini). J Hum Evol 2004, 46(2):223-237.

15. Dutrillaux B, Couturier J, Chauvier G: Chromosomal evolution of 19 species of sub-species of Cercopithecinae. Ann Genet 1980, 23(3): | 33-| 43.

16. Gebo DL, Sargis EJ: Terrestrial adaptations in the postcranial skeletons of guenons. Am J Phys Anthropol 1994, 93(3):34I-37I.

17. Okada N: SINEs. Curr Opin Genet Dev I99I, I(4):498-504. 
18. Ryan SC, Dugaiczyk A: Newly arisen DNA repeats in primate phylogeny. Proc Natl Acad Sci USA 1989, 86(23):9360-9364.

19. Murata S, Takasaki N, Saitoh M, Okada N: Determination of the phylogenetic relationships among Pacific salmonids by using short interspersed elements (SINEs) as temporal landmarks of evolution. Proc Natl Acad Sci USA 1993, 90( 1 5):6995-6999.

20. Hillis DM: SINEs of the perfect character. Proc Natl Acad Sci USA 1999, 96( 18):9979-998I.

21. Shedlock AM, Okada N: SINE insertions: powerful tools for molecular systematics. Bioessays 2000, 22(2): I 48- 160.

22. Shedlock A, Takahashi K, Okada N: SINEs of speciation: tracking lineages with retroposons. Trends Ecol Evol 2004, 19(10):545-553.

23. Ray DA, Xing J, Salem AH, Batzer MA: SINEs of a nearly perfect character. Systematic Biology 2006, 55(6):928-935.

24. van de Lagemaat LN, Gagnier L, Medstrand P, Mager DL: Genomic deletions and precise removal of transposable elements mediated by short identical DNA segments in primates. Genome research 2005, I5(9): 1243-1249.

25. Batzer MA, Deininger PL: Alu repeats and human genomic diversity. Nat Rev Genet 2002, 3(5):370-379.

26. Lander ES, Linton LM, Birren B, Nusbaum C, Zody MC, Baldwin J, Devon K, Dewar K, Doyle M, FitzHugh W, et al.: Initial sequencing and analysis of the human genome. Nature 200I, 409(6822):860-92I.

27. Salem AH, Ray DA, Xing J, Callinan PA, Myers JS, Hedges DJ, Garber RK, Witherspoon DJ, Jorde LB, Batzer MA: Alu elements and hominid phylogenetics. Proc Natl Acad Sci USA 2003, I00(22): | 2787-|279|.

28. Xing J, Wang H, Han K, Ray DA, Huang CH, Chemnick LG, Stewart CB, Disotell TR, Ryder OA, Batzer MA: A mobile element based phylogeny of Old World monkeys. Mol Phylogenet Evol 2005, 37(3):872-80.

29. Ray DA, Xing J, Hedges DJ, Hall MA, Laborde ME, Anders BA, White BR, Stoilova N, Fowlkes JD, Landry KE, et al.: Alu insertion loci and platyrrhine primate phylogeny. Mol Phylogenet Evol 2005, 35(I): $117-126$.

30. Schmitz J, Ohme M, Zischler H: SINE insertions in cladistic analyses and the phylogenetic affiliations of Tarsius bancanus to other primates. Genetics 200I, I 57(2):777-784.

31. Roos C, Schmitz J, Zischler H: Primate jumping genes elucidate strepsirrhine phylogeny. Proc Natl Acad Sci USA 2004, I0I(29): I0650-10654.

32. Waddell PJ, Kishino $H$, Ota R: A phylogenetic foundation for comparative mammalian genomics. Genome Inform Ser Workshop Genome Inform 200 I, 12:14I-I54.

33. Tosi AJ, Buzzard PJ, Morales JC, Melnick DJ: Y-chromosomal window onto the history of terrestrial adaptation in the Cercopithecini. In The Guenons: Diversity and Adaptation in African Monkeys Edited by: Glenn ME, Cords M. New York: Kluwer Academic Publishers; 2002:15-26.

34. Takahashi K, Terai Y, Nishida M, Okada N: Phylogenetic relationships and ancient incomplete lineage sorting among cichlid fishes in Lake Tanganyika as revealed by analysis of the insertion of retroposons. Mol Biol Evol 200I, I 8(I I):2057-2066.

35. Raaum RL Sterner KN, Noviello CM, Stewart CB, Disotell TR: Catarrhine primate divergence dates estimated from complete mitochondrial genomes: concordance with fossil and nuclear DNA evidence. J Hum Evol 2005, 48(3):237-257.

36. Detwiler KM: Hybridization between Red-tailed Monkeys (Cercopithecus ascanius) and Blue Monkeys (C. mitis) in East African Forests. In The Guenons: Diversity and Adaptation in African Monkeys Edited by: Glenn ME, Cords M. New York: Kluwer Academic Publishers; 2002:79-97.

37. NIH Intramural Sequencing Center [http://www.nisc.nih.gov]

38. Kent W]: BLAT - the BLAST-like alignment tool. Genome research 2002, I 2(4656-664 [http://genome.ucsc.edu].

39. Hedges DJ, Callinan PA, Cordaux R, Xing J, Barnes E, Batzer MA: Differential alu mobilization and polymorphism among the human and chimpanzee lineages. Genome research 2004, I 4(6): $1068-1075$.

40. Rozen S, Skaletsky HJ: Primer3 on the WWW for general users and for biologist programmers. Bioinformatics Methods and Protocols: Methods in Molecular Biology 2000:365-386 [http:// frodo.wi.mit.edu/cgi-bin/primer3/primer3 www.cgi]. Totowa, NJ: Humana Press
4I. Altschul SF, Gish W, Miller W, Myers EW, Lipman DJ: Basic local alignment search tool. J Mol Biol 1990, 215(3403-4I0 [http:// www.ncbi.nlm.nih.gov/blast/].

42. Batzer Lab: Laboratory of Comparative Genomics [http:// batzerlab.Isu.edu]

43. Swofford DL: PAUP*. Phylogenetic Analysis Using Parsimony (*and Other Methods). Version 4.0b I0. 4.0bl0 edition. Sunderland, Massachusetts: Sinauer Associates, Inc; 2003.
Publish with BioMed Central and every scientist can read your work free of charge

"BioMed Central will be the most significant development for disseminating the results of biomedical research in our lifetime. "

Sir Paul Nurse, Cancer Research UK

Your research papers will be:

- available free of charge to the entire biomedical community

- peer reviewed and published immediately upon acceptance

- cited in PubMed and archived on PubMed Central

- yours - you keep the copyright

Submit your manuscript here:

http://www.biomedcentral.com/info/publishing_adv.asp
BioMedcentral 